\title{
A novel design of piezo-driven dual-dimension optical scanning mechanism
}

\author{
S. H. Chang ${ }^{\text {a) }}$ and Y. C. Tung \\ Department of Mechanical Engineering, National Taiwan University, Taipei, Taiwan, Republic of China
}

(Received 26 November 1997; accepted for publication 15 May 1998)

\begin{abstract}
An optical scanning mechanism capable of dual-dimension scanning is presented. The mechanism uses a piezoelectric element to drive a $\mathrm{V}$-shaped reflecting mirror surface through two pairs of elastic beams. The design entails the use of various vibration modes to reflect the incident light in orthogonal directions. The design, fabrication, and mechanical, electrical, and optical performance of this scanning mechanism are presented. The scanning sensitivities of 88.8 and $19.3 \mu \mathrm{rad} / \mathrm{V}$ in each scanning direction are measured when operated at frequencies of 23.8 and $48.8 \mathrm{kHz}$, respectively. The capability of fine scanning sensitivity, high operating frequency, and wide scanning range is applicable for fine adjustment of the laser beam. (C) 1998 American Institute of Physics. [S0034-6748(98)02308-9]
\end{abstract}

\section{INTRODUCTION}

Recently, high speed optical scanning mechanisms ${ }^{1}$ have been playing an important role in precision equipment. Potential applications include scanning for image recording in the laser writer and laser printer, raster scanning of optical beams for video signal generation in video television or projection systems, read/write operation in optical storage devices, mechanical and infrared imaging systems, and micro machining. These applications require high speed, high repeatability, high resolution, low optical loss and distortion, and miniature package.

The deformable mirror type ${ }^{2}$ optical scanner can be activated by using electromechanical conversion devices such as piezoelectric, polyvinylidene fluoride (PVDF) film, or electrostrictive actuators. Unique features have been claimed for each material. For example, PVDF has the advantages of thin thickness, large and arbitrary area, small density and flexibility. However, lack of strong actuation force is its main disadvantage. Electrostrictive materials, such as the relaxor type, PMN, has been demonstrated for large strain, high repeatability, and small thermal expansion. Recently, the improvements of multilayer piezoelectric ceramic provide the actuator with the larger displacement sensitivity and the lower applied voltage that is suitable for fine actuation applications such as in electro-optical components.

Fowler and Schlafer ${ }^{2}$ reviewed several methods of optical beam deflection and scanning techniques, and discussed the design criteria in which a shear type piezoelectric transducer was used to develop a reflection scanner with a bandwidth of up to $17 \mathrm{kHz}$. Steinhaus and Lipson ${ }^{3}$ described a bimorph deformable mirror to correct the atmospheric turbulence aberrations for the telescope. The surface profile of the mirror constructed from laminating a glass plate and a piezoelectric ceramic can be varied by the control of the deforma-

${ }^{a)}$ Electronic mail: shchang@ccms.ntu.edu.tw tion of the laminate plate via a series of electrodes. Gomi et $a .^{4}{ }^{4}$ reported a bistable optical device using the electrostrictive displacement transducer in stabilizing the optical output power of the light source. PVDF film actuators were multilayered to construct a single dimensional deformable mirror. ${ }^{5,6}$ Again, the control of the mirror surface was achieved by the supply voltage to the PVDF. Okuda et al. ${ }^{7}$ developed a laser scanner using two longitudinally stacked piezoelectric actuators to achieve optical scanning in $x$ and $y$ directions. The scanner with dimension of $50 \times 50$ $\times 100 \mathrm{~mm}^{3}$ was driven at voltage up to $400 \mathrm{~V}$ and achieved the sensitivity of up to $0.01 \mathrm{mrad} / \mathrm{V}$ with a bandwidth of 1.0 $\mathrm{kHz}$. In the case of measurements, Cunningham et al. ${ }^{8}$ described a reflecting mirror attached to the end of a small cantilever to detect vibrations using a quadrant detector with a laser beam operating at the resonant frequency of $1.4 \mathrm{kHz}$.

In the above literature, only the scanner in Ref. 7 is capable of two-dimensional scanning. The two-dimensional scanner may be constructed by combining the onedimensional deformable mirror described in Refs. 1-6. In this article, we report a two-dimensional optical scanning mechanism driven by a single piezoelectric actuator and operated at high speed. The scanning mechanism is to deflect a narrow laser beam from an external light source. The design of this mechanism is made possible by parametric analysis based on finite element modeling. Two prototypes with different dimensions were designed and fabricated for comparison. The dynamic characteristics of the scanner were evaluated by electrical and mechanical measurements. In the experiment, a laser, whose light was measured by a quadrant detector, was used to observe the optical scanning performance. All the experimental data were compared with theoretical results to investigate the validity of the analysis. The developed scanning mechanism has the merit of high resolution, high speed, and small package that will be suitable for applications described above. 


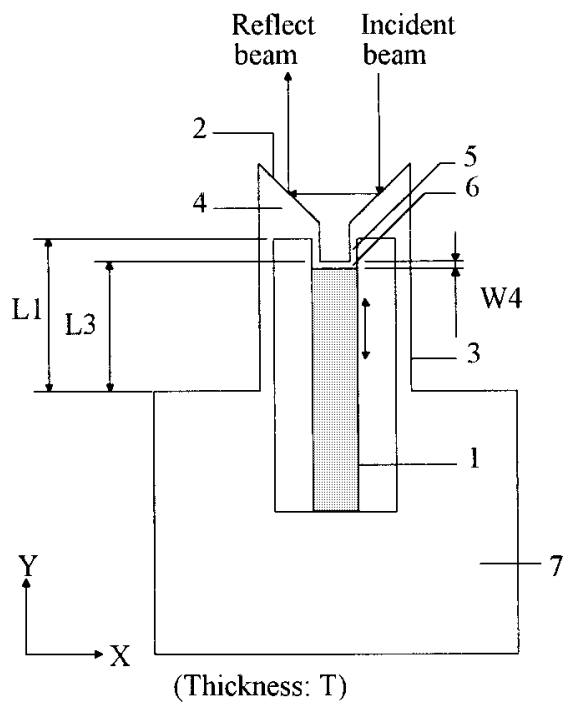

FIG. 1. Design of the scanning mechanism with the key dimensions and the coordinate system. (1) Piezoelectric actuator, (2) reflecting surface, (3) support beam, (4) triangular mass, (5) drive beam, (6) drive plate, (7) base plate.

\section{DESIGN AND ANALYSIS}

\section{A. Basic mechanism}

The design of the scanning mechanism, shown in Fig. 1, includes a piezoelectric actuator, two $\mathrm{V}$-shaped reflecting surfaces on the triangular mass, two support beams, and a drive plate connecting the two drive beams. The principle of the design is described as follows. When the scanner is operated by exciting the piezoelectric actuator at the resonant frequency, the reflecting surfaces oscillate at the associate vibration modes, thus causing the incident light to be reflected and allow the mechanism to perform the scan. In this design, the capability of dual-dimension scanning is made possible by the resonant vibration occurring at the two distinct vibration modes of the scanner. The excitation of the resonant vibration is provided by the electrically induced piezoelectric motion. The design of the symmetric geometry allows the total reflecting angle to be doubled by the symmetric deformation of the mechanism. When the reflecting surface is rotated by an angle of $\theta$, the incident light, shown by a single arrow in Fig. 1 , is reflected by $2 \theta$ at each Vshaped reflecting surface. Therefore, the incident light is allowed to be reflected twice, resulting in a total reflect angle of $4 \theta$. With the tight close loop mechanical design, the scanner can be operated at a high resonant frequency. This design also reduces the geometric errors caused by the thermal drift.

The multilayer piezoelectric ceramic element serves as an actuator to provide the force and displacement to the mechanism. In response to an applied voltage, the piezoelectric actuator, using longitudinal mode $\left(d_{33}\right)$ of its piezoelectric effect, oscillates longitudinally in the $Y$ direction as shown by the double arrow in Fig. 1. Following the motion of the actuator, the drive plate excites the drive beams, the triangular masses, and the support beams.

A way to control the dual-dimension of the scanner is through the use of the resonant vibrations operating at different modes. The vibration modes suitable for scanning in-

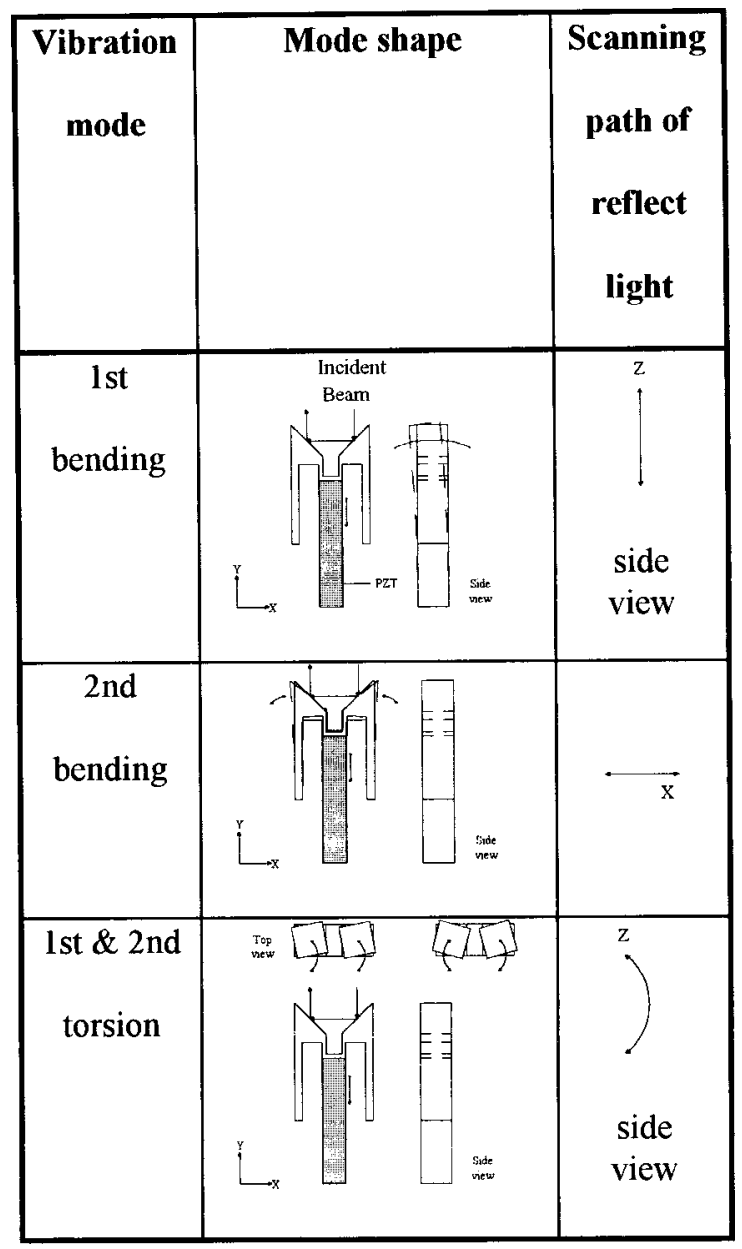

FIG. 2. The vibration modes of the scanning mechanism used to reflect the light beam.

clude the first and second bending, and the first and second torsion modes. Figure 2 illustrates these vibration modes, and the corresponding scanning path of the reflected light shown in the coordinate system. In this study, only the bending modes were used for optical scans in the $X$ and $Z$ directions. The torsion modes are also good for the scan in the $X-Z$ plane, and the reflected beam is along an arc path.

\section{B. FEM analysis}

The primary requirements for this scanner are high speed, and a wide scanning range. A critical need of the overall design is to separate the resonant frequencies far from each other to avoid the coupling effect between these modes. In order to satisfy the requirement of modal decoupling of the scanning mechanism, the dominant parameters of the mechanism $L 1, L 3, W 4$, and $T$ (as shown in Fig. 1) were extensively studied to observe the influence.

The finite element method (FEM) is the most extensive technique used to simulate the mechanical behavior, particularly for structures with complex geometry and different materials. In this study, a FEM software, ANSYS, was used for modal analysis of the scanner. For clear observation of the mode shape, three-dimensional structure elements were used to construct the finite element model. The electromechanical 

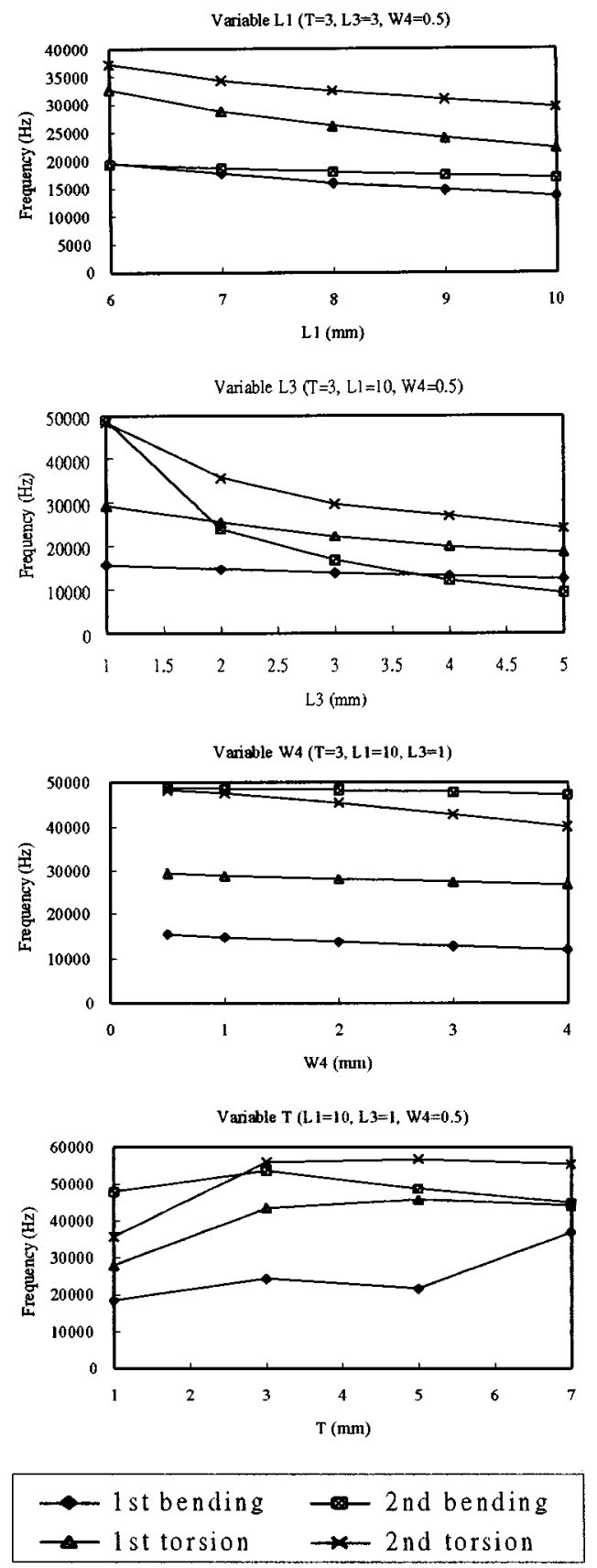

FIG. 3. The computed resonant frequency for different vibration modes at dependence of geometry parameters $L 1, L 3, W 4$, and $T$.

coupling element was used to simulate the electrical and mechanical behavior of the piezoelectric actuator.

By using FEM, the resonant frequencies and the corresponding mode shapes of the mechanism were computed and identified in detail. The computed results of the parametric analysis for the dominant parameters are presented in Fig. 3.

Figure 3 shows the sensitivity of each parameter to the variation of the resonant frequency at each vibration mode. For example, parameter $W 4$ is not sensitive to vibration modes except for the second torsion mode. On the contrary, parameter $L 3$ is more sensitive than others, especially for the second bending mode. By carefully choosing the dimension of each parameter, the frequency of each mode was tuned and well separated between modes.
TABLE I. The dimensions of two prototypes, DESIGN1 and DESIGN2 (unit: $\mathrm{mm}$ ).

\begin{tabular}{lcccccc}
\hline & $L 1$ & $L 3$ & $W 4$ & $T$ & $\begin{array}{c}\text { Piezoelectric } \\
\text { transducer }\end{array}$ & $\begin{array}{c}\text { Total } \\
\text { dimension }\end{array}$ \\
\hline DESIGN1 & 6 & 1 & 3 & 3 & $2 \times 3 \times 10$ & $5 \times 8.5 \times 3$ \\
DESIGN2 & 10 & 3 & 0.5 & 3 & $2 \times 3 \times 10$ & $5 \times 12.5 \times 3$ \\
\hline \hline
\end{tabular}

From the analysis of the mechanism, the influence of these dominant parameters on the resonant frequency and the corresponding mode shapes was observed. Dimensions of two prototype designs named DESIGN1 and DESIGN2 were determined and shown in Table I.

\section{EXPERIMENT}

\section{A. Fabrication}

Based on the analysis results described above, two prototypes of the experimental mechanism were fabricated. Fabricating the scanning mechanism involved two steps: machining the base plates and elastic beams from a metal blank, and installing the piezoelectric actuator in the scanning mechanism. The scanning mechanism itself was machined from a $3 \mathrm{~mm}$ thick 6061-T6 aluminum blank.

The multilayer piezoelectric actuator with dimension of $2 \times 3 \times 10 \mathrm{~mm}$ was used to provide the force and displacement. It is capable of providing a maximum displacement of $9.1 \mu \mathrm{m}$ at $150 \mathrm{vdc}$, generating the force of $200 \mathrm{~N}$ and has the resonant frequency of $138 \mathrm{kHz}$.

In order to prevent the degenerated vibration modes in this complex structure, the geometry and its dimension of the mechanism has to be precisely maintained. Mechanical components such as the support beams and the drive beams, having an especially strong influence on the resonance of the scanning mechanism, are critically important. Due to their strong effects, both the dimensional tolerance and the geometric tolerance are critical to the performance. To assure the proper qualities, the mechanism was fabricated by precision wire electrodischarge machining.

The installation of the piezoelectric actuator was equally important. The adhesion between the piezoelectric actuator and the mechanism should be complete over all the contact surface. The flatness and parallelism of the surface where the actuator was installed should be maintained precisely. The

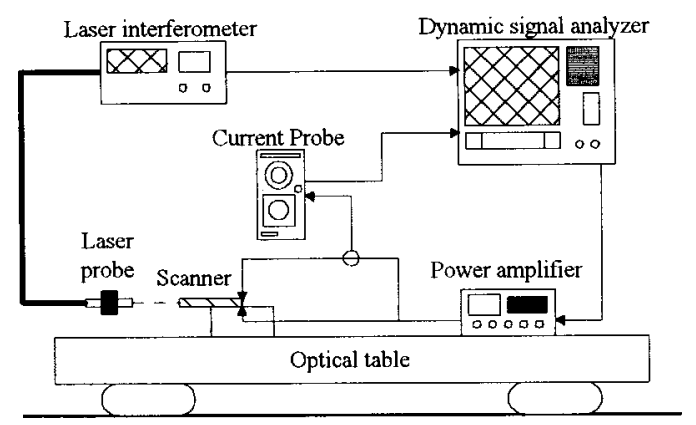

FIG. 4. Experimental setup for mechanical and electrical measurement of the scanning mechanism. 


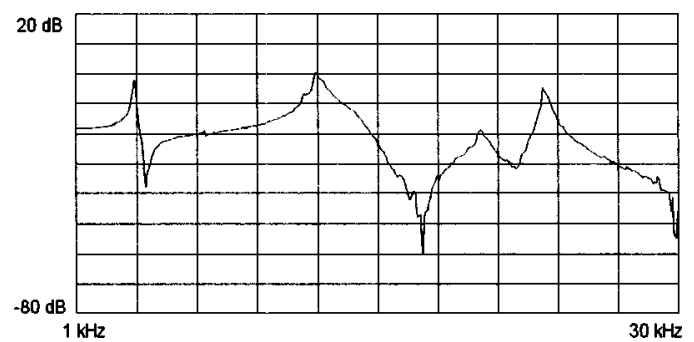

FIG. 5. Frequency response function of prototype DESIGN2 in the $X$ direction using displacement measurement.

actuator also has to be positioned precisely to avoid exerting the lateral force on the mechanism.

The experimental scanner prototypes were fabricated. Their performance was experimentally evaluated by mechanical, electrical, and optical measurements.

\section{B. The mechanical measurement}

Mechanical measurements were used to investigate the displacement sensitivity, the resonant frequencies, and the vibration modes of the scanner. The displacement sensitivity was measured by using a Polytec laser interferometer which has the measuring resolution of $8 \mathrm{~nm}$ and a bandwidth of 50 $\mathrm{kHz}$. The optical probe of the laser interferometer was placed pointing at different positions of the scanner to investigate the resonant frequencies and the corresponding vibration modes. The vibration source was the displacement of the piezoelectric actuator in response to an electrical voltage, which was generated from a dynamic signal analyzer. The displacement data measured by the laser interferometer were transformed into the frequency response function by a dynamic signal analyzer. The experimental setup is shown schematically in Fig. 4.

One of the measured frequency response functions in the $X$ direction measured by the dynamic signal analyzer is shown in Fig. 5. From the measured frequency response functions, the resonant frequencies can then be found and the

TABLE II. The resonant frequencies of prototype DESIGN1 for different vibration modes obtained from the experiments and FEM analysis.

\begin{tabular}{lccccc}
\hline \hline & \multicolumn{5}{c}{ Resonant frequency (Hz) } \\
& & $\begin{array}{c}\text { First } \\
\text { bending }\end{array}$ & $\begin{array}{c}\text { Second } \\
\text { bending }\end{array}$ & $\begin{array}{c}\text { First } \\
\text { torsion }\end{array}$ & $\begin{array}{c}\text { Second } \\
\text { torsion }\end{array}$ \\
\hline $\begin{array}{l}\text { FEM analysis } \\
\begin{array}{l}\text { Mechanical } \\
\text { measurement }\end{array}\end{array}$ & $(b)$ & 19707 & 50977 & 37018 & 44601 \\
$\begin{array}{l}\text { Electrical } \\
\text { measurement }\end{array}$ & $(c)$ & 23800 & 48800 & 34200 & 44700 \\
& $\frac{(b)-(a)}{(a)}$ & 20.769 & -4.271 & -7.613 & 0.222 \\
$\begin{array}{l}\text { Difference } \\
(\%)\end{array}$ & $\frac{(c)-(a)}{(a)}$ & 21.657 & -4.859 & -2.412 & -0.507 \\
& $\frac{(c)-(b)}{(b)}$ & 0.735 & -0.615 & 5.629 & -0.727 \\
\hline \hline
\end{tabular}

TABLE III. The resonant frequencies of prototype DESIGN2 for different vibration modes obtained from the experiments and FEM analysis.

\begin{tabular}{|c|c|c|c|c|c|}
\hline \multicolumn{6}{|c|}{ Resonant frequency $(\mathrm{Hz})$} \\
\hline & & $\begin{array}{c}\text { First } \\
\text { bending }\end{array}$ & $\begin{array}{l}\text { Second } \\
\text { bending }\end{array}$ & $\begin{array}{l}\text { First } \\
\text { torsion }\end{array}$ & $\begin{array}{l}\text { Second } \\
\text { torsion }\end{array}$ \\
\hline \multirow{6}{*}{$\begin{array}{l}\text { FEM analysis } \\
\text { Mechanical } \\
\text { measurement } \\
\text { Electrical } \\
\text { measurement }\end{array}$} & (a) & 13507 & 16692 & 22089 & 29473 \\
\hline & (b) & & & & \\
\hline & & 12393 & 16584 & 23540 & 32237 \\
\hline & (c) & & & & \\
\hline & & 12960 & 15025 & 22788 & 33193 \\
\hline & $\frac{(b)-(a)}{(a)}$ & -8.248 & -0.647 & 6.569 & 9.378 \\
\hline \multirow[t]{2}{*}{$\begin{array}{l}\text { Difference } \\
(\%)\end{array}$} & $\frac{(c)-(a)}{(a)}$ & -4.050 & -9.987 & 3.164 & 12.622 \\
\hline & $\frac{(c)-(b)}{(b)}$ & 4.575 & -9.401 & -3.195 & 2.966 \\
\hline
\end{tabular}

corresponding vibration modes can also be identified by the typical modal testing procedure. The resonant frequencies of vibration modes obtained from the experiments and FEM analysis are listed in Tables II and III. The difference between results is also listed as a percentage.

\section{The electrical measurement}

Electrical measurements were then used to further investigate the resonant and anti-resonant frequencies of the electrical system of the scanning mechanism. Using a Tektronix current probe, the electric current passing through the piezoelectric actuator during the swept sine excitation was measured. The resonant and anti-resonant frequencies were found where the local maximum and minimum, respectively, of the current existed. The experimental setup is shown schematically in Fig. 4.

For the conversion efficiency between electrical and mechanical energy of the piezoelectric actuator, the electromechanical coupling coefficient (EMCC) is an important index. Mason ${ }^{9}$ described the EMCC as

$$
k_{d}^{2}=\frac{f_{a}^{2}-f_{r}^{2}}{f_{a}^{2}},
$$

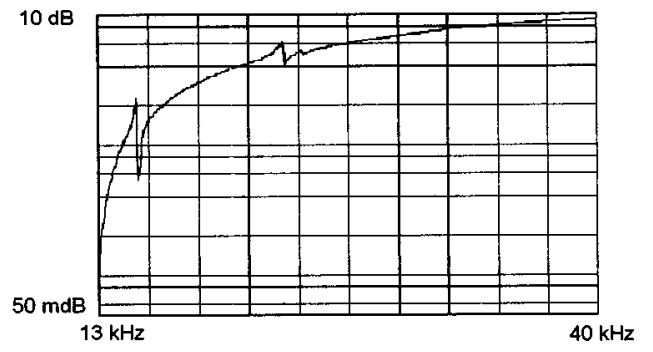

FIG. 6. Frequency response function of prototype DESIGN2 using electric current measurement. 


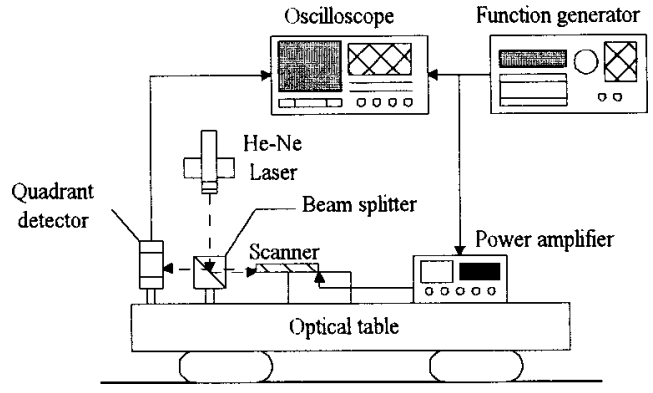

FIG. 7. Experimental setup for optical measurement of the scanning mechanism.

where $f_{r}$ is the resonant frequency, and $f_{a}$ is the antiresonant frequency of the piezoelectric actuator.

The frequency response function obtained from the electrical measurement of the piezoelectric actuator in the scanning mechanism is shown in Fig. 6. Tables II and III, respectively, list the resonant frequencies of the two designs obtained from the experimental measurements, and the difference between the experimental and FEM analysis values.

From the resonant and anti-resonant frequencies of the first bending modes, the electromechanical coupling coefficients of DESIGN1 and DESIGN2 were calculated to be 0.364 and 0.342 , respectively. In addition, the phenomenum that the conductivity tended to increase when a higher excitation frequency was applied to the piezoelectric actuator was observed in experiments. That is due to the fact that the piezoelectric actuator plays a role of capacitor in the equivalent electric circuit.

\section{The optical measurement}

Finally, the scanning performance of the scanning mechanism was evaluated by optical measurements. The positions of the reflect laser beam that were projected to a plane parallel to the $X-Z$ plane were dynamically measured by a $0.1 \mu \mathrm{m}$ resolution quadrant detector. The experimental setup is depicted schematically in Fig. 7. The light source was a 15 $\mathrm{mW} \mathrm{He}-\mathrm{Ne}$ laser. The calibration of the quadrant detector was conducted prior to the optical measurements, in order to correlate the given displacement of the light beam with detector output electrical voltage.

The calibration was done by attaching the quadrant detector to a three-dimensional micrometer stage. The detector

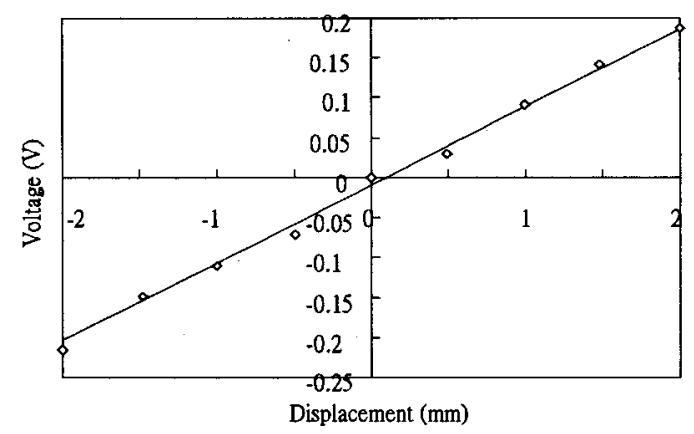

FIG. 8. Calibration of the quadrant detector.

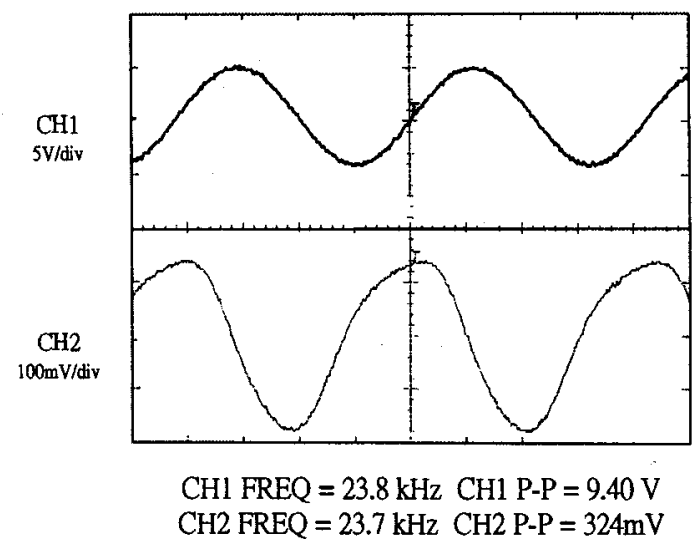

FIG. 9. The input signal (upper curve) and the output signal (lower curve) from the quadrant detector in $Z$ direction, while driving the piezoelectric actuator in DESIGN1 at $23.8 \mathrm{kHz}$.

was moved in one direction perpendicularly to the stationary incident laser beam. The readings from the micrometer and from the oscilloscope were recorded and plotted as shown in Fig. 8. Then the coefficient converting the detector's voltage to displacement can be calculated using the least square method. In this experiment the conversion coefficient of the quadrant detector was $0.1007 \mathrm{~mm} / \mathrm{V}$. By this coefficient, the displacement of the reflect beam projected on the quadrant detector can be found.

The optical measurements of the scanner were conducted under the conditions operated at its first two bending modes. The range of the scanning beam was measured by recording the positions of the light spot detected by the quadrant detector. Figure 9 shows the recorded input signal from the function generator and the output signal from the quadrant. From the output data of the quadrant detector, the sensitivity of the reflected angle of the scanner was then calculated. Table IV lists the amplitude of the input signal, the corresponding reflect angle, and sensitivity, which were obtained from the experiments.

\section{DISCUSSION}

Comparing the results in Tables II and III, a general agreement between analytical and experimental results can be found except for the first bending mode of DESIGN1.

TABLE IV. The measured performance of the prototype DESIGN1.

\begin{tabular}{lcc}
\hline \hline \multicolumn{1}{c}{ Mode } & 1st bending & 2nd bending \\
\hline $\begin{array}{l}\text { Input } \\
\left(V_{\mathrm{p}-\mathrm{p})}\right)\end{array}$ & 100 & 100 \\
$\begin{array}{l}\text { Operating } \\
\text { frequency }(\mathrm{Hz})\end{array}$ & 23800 & 48800 \\
$\begin{array}{l}\text { Reflect angle } \\
(\text { (mrad) }\end{array}$ & 8.88 & 1.93 \\
$\begin{array}{l}\text { Reflect angle } \\
\text { sensitivity } \\
(\mu \mathrm{rad} / \mathrm{V})\end{array}$ & 88.8 & 19.3 \\
\hline
\end{tabular}


This may be due to the improper installation of the piezoelectric actuator, which causes unpredictable force to be exerted on the mechanism, resulting in a variation in the resonant frequency. It also may have been due to the material properties of the piezoelectric element.

We have described the novel design of a dual-dimension optical scanning mechanism using a single piezoelectric actuator. This scanner was designed, fabricated, and evaluated experimentally. The study demonstrates the compact package, the high sensitivity, and the high speed. The deflecting angle measured $8.88 \mathrm{mrad}$ at $23.8 \mathrm{kHz}$ resonant frequency for one direction, and $1.93 \mathrm{mrad}$ at $48.8 \mathrm{kHz}$ resonant frequency for the orthogonal direction. The capability is practical for the optical inspection applications, and particularly suited for fine adjustment of the laser beam.

\section{ACKNOWLEDGMENT}

This work was supported by the National Science Council, Taiwan, Republic of China, under Grant No. NSC862221-E-002-096.

${ }^{1}$ G. F. Marshall, Optical Scanning (Marcel Dekker, New York, 1991).

${ }^{2}$ V. J. Fowler and J. Schlafer, Appl. Opt. 5, 1675 (1966).

${ }^{3}$ E. Steinhaus and S. G. Lipson, J. Opt. Soc. Am. 69, 478 (1979).

${ }^{4}$ M. Gomi, K. Uchino, M. Abe, and S. Nomura, Jpn. J. Appl. Phys., Part 2 20, L375 (1981).

${ }^{5}$ T. Sato, H. Ishida, and O. Ikeda, Appl. Opt. 19, 1430 (1980).

${ }^{6}$ T. Sato, H. Ishikawa, and O. Ikeda, Appl. Opt. 20, 3664 (1982).

${ }^{7}$ M. Okuda, N. Wakita, K. Ohya, H. Banno, and S. Hattori, J. Appl. Phys. 25, 223 (1986).

${ }^{8}$ M. J. Cunningham, D. F. L. Jenkins, W. W. Clegg, and M. M. Bakush, Sens. Actuators A 50, 147 (1995).

${ }^{9}$ W. P. Mason, Piezoelectric Crystals and their Application to Ultrasonics (Van Nostrand, New York, 1950). 